\title{
The task pre-configuration is associated with cognitive performance evidence from the brain synchrony
}

Chanjuan Fan ( $\nabla 1204094246 @ q q . c o m$ )

Taiyuan University of Technology https://orcid.org/0000-0002-7371-9732

Jing Wei

Taiyuan University of Technology

Jie Xiang

Taiyuan University of Technology

Ying Li

Taiyuan University of Technology

Lan Yang

Taiyuan University of Technology

Jiaqi Lv

Taiyuan University of Technology

Hao Guo

Taiyuan University of Technology

\section{Research Article}

Keywords: synchrony, high-order cognitive networks, the task-general architecture, update efficiency

Posted Date: November 5th, 2021

DOI: https://doi.org/10.21203/rs.3.rs-1023537/v1

License: (9) This work is licensed under a Creative Commons Attribution 4.0 International License.

Read Full License 


\section{The task pre-configuration is associated with cognitive performance}

\section{evidence from the brain synchrony}

Chanjuan Fan ${ }^{1}$, Jing Wei ${ }^{1}$, Jie Xiang ${ }^{1}$, Ying Li $^{1}$, Lan Yang ${ }^{1}$, Jiaqi Lv ${ }^{1}$, and Hao Guo ${ }^{1, *}$ ${ }^{1}$ Taiyuan Univ Technol, Coll Informat \& Comp, Taiyuan 030024, Peoples R China

* Taiyuan Univ Technol, Coll Informat \& Comp, Taiyuan 030024, Peoples R China 1204094246@qq.com (Chanjuan Fan); 20141032@sxufe.edu.cn (Jing Wei); xiangjie@tyut.edu.cn (Jie Xiang); liying01@tyut.edu.cn (Ying Li);

yanglan0333@link.tyut.edu.cn (Lan Yang); 1vjiaqi0393@link.tyut.edu.cn (Jiaqi Lv);

${ }^{\text {* } C o r r e s p o n d e n c e: ~ f e i y u \_g u o @ s i n a . c o m ~(H a o ~ G u o) ; ~ T e l: ~+86-18636635818 ~}$

Word counts for the abstract: 214

Word counts for manuscript body: 3078 


\section{Abstract}

Although many characteristics of the resting state and task states have been studied, it is still unclear how the brain network switches from the resting state during tasks. The current theory is that the brain is a complex dynamic system and synchrony is defined to measure brain activity. This study compared the changes in synchrony between the resting state and different task states in healthy young participants and examined the ability to switch from the resting state to the task-general architecture of synchrony $(\mathrm{N}=954)$. We found that the synchrony increased significantly during the tasks and the brain has the task-general architecture of synchrony during different tasks, the increase of synchrony in high-order cognitive networks is particularly obvious, while the increase in the sensorimotor network is relatively low. In addition, the high synchrony of high-order cognitive networks in the resting state can promote effective task switching and the pre-configured participants have better cognitive performance, which shows that the spontaneous brain activity and cognitive ability are closely related. These results revealed changes in the configuration of the brain network for switching between the task states and the resting state, highlighted the consistent changes in the brain network between different tasks, and found that there is an important relationship between switching ability and cognitive performance.

Keywords: synchrony, high-order cognitive networks, the task-general architecture, update efficiency.

\section{Introduction}

The resting and task state fMRI is widely used for non-invasive assessment of functional brain activity, and recent developments have led to many studies describing the relationship between task state and resting state regions (Dajani et al., 2020; Freitas et al., 2020; Friston, 2011). Recent research has found that global information transmission and the integration of resting-state networks (RSNs) will be more efficient 
during the mission. The economic theory based on brain network organization indicates that the brain network should be in a resting state in an energy-saving mode, and at the same time showed a dynamic network reorganization under task requirements in order to promote the transmission of information between network and network(AvenaKoenigsberger, Misic, \& Sporns, 2017; Bullmore \& Sporns, 2012). However, most of them are based on the study of static properties such as functional connections and network topology (Markett, Jawinski, Kirsch, \& Gerchen, 2020; Zhang, Tang, Zhou, \& Li, 2020), how the brain network coordinates to express cognitive operations in dynamic properties is still unclear.

Current studies have confirmed that the brain is a complex dynamic system (Kringelbach \& Deco, 2020; Vasa et al., 2015), so our understanding of the brain should be further developed from a dynamic perspective research. The synchrony is an important feature of the nonlinear dynamics of the brain. In the brain, the communication between different brain regions should take place through coherence, in which two brain regions with synchronous fluctuations of activity can exchange information (Fries, 2005).

Some recent studies advocated a more general architecture between tasks, while other studies advocated differentiation between tasks. We believed that a general architecture between multiple tasks would greatly simplify the research of functional brain organization-from the need to consider almost infinitely diverse task states to considering a single (or a few) network architecture with severely constrained state space. Studies have confirmed that the brain has a general architecture of functional interconnection networks among various tasks, and the functional network architecture during task execution is mainly a resting state network architecture caused by changes in general tasks and task-specific networks (Schultz \& Cole, 2016a, 2016b).

We assumed that there was also a task-general architecture of synchrony during the tasks, and we tried to calculate the synchronicity of interaction between different RSNs, explore the differences between resting state and different tasks, and seek the commonalities of these changes. Finally, we studied the switching ability of synchrony from resting state to task-general architecture and explored the relationship with 
synchrony and cognitive performance, including task accuracy and fluid intelligence and crystallized intelligence.

\section{Methods}

The overall experimental design was shown in Fig.1. The BOLD signal was extracted from the pre-processed image, and filtered the extracted BOLD signal. Then the BOLD signal was converted into phase through Hilbert transform, and synchrony was calculated (Fig. 1A). Subsequently, the synchrony interaction matrix of resting state and task states was verified by NBS, and the task-general architecture of synchrony was obtained by PCA. Finally, a linear regression was performed for update efficiency and synchronization, as well as cognitive performance (Fig. 1B).

\subsection{Participants}

The data were collected by the Washington University-Minnesota Consortium Human Connectome Project (Van Essen et al., 2013). The data come from the "S1200 Subject" release (see https://www.humanconnectome.org/data ). A total of 954 participants were selected for this study. See Table 1 for basic information about the participants. Due to the lack of accuracy of gambling, social and motor behaviors, only the results of working memory, language, emotion and relationship are considered. All participants were assessed for a history of neurological and psychiatric disorders, psychotropic drug use, and physical condition or implants.

\subsection{Data and data pre-processing}

Whole-brain echoplanar scans were acquired with a 32 channel head coil on a modified 3T Siemens Skyra with TR $=720 \mathrm{~ms}, \mathrm{TE}=33.1 \mathrm{~ms}$, flip angle $=52^{\circ}, \mathrm{BW}=$ $2290 \mathrm{~Hz} / \mathrm{Px}$, in-plane FOV $=208 \times 18 \mathrm{~mm}$, 72slices, $2.0 \mathrm{~mm}$ isotropic voxels, with a multiband acceleration factor of 8 (Ugurbil et al., 2013). In addition to the resting state, there were seven in-scanner tasks designed to engage a variety of cortical and subcortical networks related to emotion perception, relational reasoning, language 
processing, working memory, gambling, social cognition and motor responses. Table 2 briefly introduces these seven tasks. These tasks are described in more detail in (Barch et al., 2013).

We used a minimally pre-processed version of the data that included spatial normalization to a standard template, motion correction, slice timing correction, intensity normalization and surface and parcel constrained smoothing of $2 \mathrm{~mm}$ full width at half maximum (Glasser et al., 2013). Filtering 0.06-0.125 Hz was applied to the data, which is thought to be especially sensitive to dynamic changes in task-related functional brain (Han et al., 2017). Since each task contains two runs, in order to avoid the impact of insufficient time on the experiment, both runs are connected to a BOLD signal. The first ten time points and the last ten time points were then removed to minimize the boundary effect (Ponce-Alvarez et al., 2015). To facilitate comparison between rest and task-based conditions, both sets of data were identically processed.

\subsection{Definition of RSNs from functional imaging data}

Extracted the BOLD signal from the Glasser 36 atlas and use the BOLD signal for regional analysis. Using multimodal segmentation of the human cerebral cortex (Glasser et al., 2016), 360 brain regions were assigned to 14 RSNs, including visual network (VIS), somatomotor network (SMN), cingulo-opercular network (CON), premotor1 (PREM1), premotor2 (PREM2), default mode network (DMN), frontoparietal network (FPN), primary auditory (AUD), language (LAN), posterior cingulate (PCC), hippocampal (HIPP), posterior multimodal (PMULTI) and uncertain (NONE).

\subsection{Calculate the synchrony}

The synchrony was used to describe the "instantaneous" collective behavior of a group of phase oscillators (Skardal \& Arenas, 2020). First, converted the BOLD signal into a complex phase through the Hilbert transform. The calculation formula for synchrony is as follows: 


$$
\text { Synchrony }=\frac{1}{N T}\left|\sum_{t=1}^{T} \sum_{n=1}^{N} e^{i \theta_{n}(t)}\right|
$$

where $\Theta_{n}(t)$ is the instantaneous phase of oscillator $\mathrm{n}$ at time $\mathrm{t}, \mathrm{T}$ is the number of time points. For global synchrony, $\mathrm{N}$ is the number of brain regions. For synchrony between RSNs, each interaction involves two RSNs, where $\mathrm{N}$ refers to the number of brain regions included in the two RSNs. With complete independence, all phases were uniformly distributed and synchrony approaches zero. Conversely, if all phases are equivalent, synchrony approaches one (Bakhshayesh, Fitzgibbon, Janani, Grummett, \& Pope, 2019).

\subsection{Network-based statistic}

The NBS is a non-parametric statistical test designed to deal with the multiple comparisons problem in a graph by identifying the largest connected sub-component in topological space while controlling the family wise error rate. An "interaction matrix" was estimated for each subject, reflecting the synchrony interaction of 14 RSNs (Alderson et al., 2018). The NBS test was performed on the resting state synchrony interaction matrix of 954 subjects and obtained the synchrony interaction matrix under different tasks, and the synchrony difference between the seven groups of resting state and task state was determined (Fig. 1B).

\subsection{Principal component analysis}

Principal Component Analysis (PCA) is the most commonly used method to reduce linear dimensionality. Its goal is to map high-dimensional data to low-dimensional space through a certain linear projection (J. Li et al., 2019). Loadings are mainly used for the importance of a variable in each principal component (PC), that is, the weight of each variable in different PCs how many (Min, Liu, \& Zhang, 2018; Petersen, Zhao, Carmichael, \& Muller, 2016). In order to quantify the level of sharing of the seven taskbased configurations, PCA was used to reduce the dimensions of the seven task-based synchrony interaction matrices to a one-dimensional matrix, retaining most of the 
characteristics of the task states (Fig. 1B). The synchrony interaction matrix of a single subject was vectorized based on the seven task states, and obtained the task architecture of the synchrony of a single subject by PCA. Subsequently, a task-general architecture of synchrony was obtained by simple average across subjects.

\subsection{Update efficiency}

Update efficiency refers to the ability to switch from a resting state into a task-based configuration (Schultz \& Cole, 2016a). Due to the high update efficiency, the network architectures of the resting state and the task state are very similar, so that almost no configuration changes are required when switching between the two. The update efficiencies were calculated for all subjects by vectorizing the upper triangular half and diagonal of the rest and task-general architecture of synchrony and calculating their Pearson's correlation coefficients. Finally, the update efficiency of all subjects was transformed to a normal distribution by Fisher-z.

\section{Results}

\subsection{Difference of global synchrony between resting state and task states}

Compared with the resting state, global synchrony during the tasks was significantly higher ( $p<0.001$, Fig. 2). These mainly include language $(t=19.01, p<0.001)$, emotion $(t=19.06, p<0.001)$, relational $(t=20.67, p<0.001)$, social $(t=23.49$, $p<0.001)$, working memory $(t=25.35, p<0.001)$, gambling $(t=25.04, p<$ $0.001)$, motor $(t=36.09, p<0.001)$.

\subsection{Difference of RSNs synchrony between resting state and task states}

During the tasks, the synchrony between RSNs generally increased, especially in high-order cognitive networks. A fixed threshold of 15 was selected to visualize the increase in synchrony between RSNs under the same scale. (Fig. 3, $p<0.001$, corrected). Fig. 3 showed the maximum connected subgraph with increased 
synchrony detected by NBS under a fixed threshold of seven tasks.

\subsection{The similarity of RSNs synchrony during the tasks}

Through PCA, there were similar results among the seven tasks. On average, the PC1 accounted for $85 \%$ of the variance (Fig. 4A). The loadings of the seven tasks were positive and evenly distributed (Fig. 4B). These included language $=0.374$, emotion $=$ 0.378 , relational $=0.390$, social $=0.316$, working memory $=0.370$, gambling $=0.382$, motor $=0.397$. The Pearson correlation was performed on the synchrony between seven tasks and found that the correlation value can reach more than 0.75 ( $p<0.01$; Fig. 4C).

\subsection{High synchrony and low synchrony between RSNs}

The task-general architecture of synchrony was decomposed into a low synchrony subnet (Fig. 5A) and a high synchrony subnet (Fig. 5B). High synchrony subnets mainly included high-order cognitive networks, while low synchrony subnets were mainly related to sensorimotor networks.

\subsection{Correlation between synchrony of RSNs and update efficiency}

Correlating the synchrony of each network connection with the update efficiency, the slope of the linear regression equation (Fig. 6A) and its significance (Fig. 6B, $p<0.01$, FDR) were obtained. RSNs showing a significant positive correlation were mainly in high-order cognitive networks (Fig. 6C), while most sensorimotor networks were negatively correlated (Fig. 6D).

\subsection{Correlation between update efficiency and cognitive performance}

Next, a linear regression analysis was performed on cognitive performance and update efficiency. There was a significant positive correlation between update efficiency and behavioral accuracy (Fig. 7A) or cognitive intelligence (Fig. 7B). Behavioral accuracy included language $(F=12.128, p=0.001)$, emotion $(F=5.500$, $p=0.019)$, relational $(F=24.073, p<0.001)$ and work memory $(F=23.232, p<$ 
$0.001)$. Cognitive intelligence included fluid intelligence $(F=13.787, p<0.001)$ and crystalline intelligence $(F=21.732, p<0.001)$.

\section{Discussion}

The research mainly explored whether there is a task-general architecture of synchrony during the tasks. We studied 954 healthy young participants. During the tasks, the synchrony was higher than the resting state (Fig. 2), especially in the high-order cognitive networks (Fig. 3). And there were similar patterns for different tasks. Then we received the task-general architecture of synchrony by PCA (Fig. 4, Fig.5). In addition, the high synchrony of high-order cognitive networks in the resting state can promote effective task switching (Fig. 6). Participants with high update efficiency had better cognitive performance (Fig. 7). Results are discussed in detail hereafter.

\subsection{There was the task-general architecture of synchrony between different tasks.}

Our results proved that there is a "task-general architecture" in synchrony (Fig. 4, Fig. 5). We reached the conclusion based on the following three results: (1) Most of the differences between tasks were resolved by PC1 (85\%); (2) The loadings were evenly distributed; (3) There was a high correlation between the seven tasks. The current results are in agreement with previous studies, the functional connection network between different tasks had the similar patterns (Chan, Alhazmi, Park, Savalia, \& Wig, 2017; Schultz \& Cole, 2016b). And metastability also showed a similar pattern for different tasks (Alderson et al., 2018). Similarities between functional network configurations that are evoked under different behavioral paradigms form a so-called 'task-general architecture"(Cole, Bassett, Power, Braver, \& Petersen, 2014). Given that the cerebral blood flow caused by different tasks accounts for less than $5 \%$ of the resting cerebral blood flow (Raichle, 2010). It also showed from the side that different tasks have similar patterns.

The experience-dependent changes in rest functional connection, demonstrating a certain plausibility for task driven mechanism (Hearne, Cocchi, Zalesky, \& Mattingley, 
2017; Millar et al., 2021). The brain's functional network architecture during task performance was shaped primarily by an intrinsic network architecture that is also present during rest, and secondarily by evoked task-general and task-specific network changes (Boring, Ridgeway, Shvartsman, \& Jonker, 2020; Messel, Raud, Hoff, Skaftnes, \& Huster, 2019; Raichle, 2010). Overall, the brain has a task-general architecture, but there are still specific task configurations in different tasks (Cole et al., 2014). This was why there are similar but not identical patterns in seven tasks.

\subsection{The synchrony of high-order cognitive networks has increased more widely during tasks}

The high-order cognitive networks had higher synchrony during the tasks, while the synchrony of the sensorimotor networks was relatively low (Fig. 2). We suspected that the result was related to the following reasons. Both high-order cognitive networks tend to have high between-RSN connectivity, indicating their roles as connector RSNs. The close connection between these RSNs and the rest of the brain could form a mechanistic explanation for their utility in myriad complex cognitive processes $(\mathrm{Gu}$, Pasqualetti, et al., 2015). In contrast to high-order cognitive networks that act as connectors, the sensorimotor networks tend to have poor between-RSN connectivity, indicating their roles as provincial RSNs. The weak connection between these RSNs and the rest of the brain indicate that they display distinct profiles of neurophysiological activity, and may perform more segregated functions (Gu, Satterthwaite, et al., 2015).

Previous studies have identified the exchange of information between regions was carried out through synchrony(M. Li et al., 2019; Palmigiano, Geisel, Wolf, \& Battaglia, 2017). And high-order cognitive networks are global hubs (Ferrier, Tiran, Deffieux, Tanter, \& Lenkei, 2020; Meijer et al., 2017), and high-order cognitive networks play a disproportionate role in shaping information transfer between regions throughout the brain(Ito et al., 2017). So the universal flexible hub networks showed higher synchrony during the mission. The high-order cognitive networks indicate that it is more flexible to transfer task information across regions and networks (Ito et al., 2017). 


\subsection{The high synchrony of the resting state high-order cognitive networks can promote task switching ability}

The high synchrony of high-order cognitive networks coupling and the low synchrony of sensorimotor network coupling can promote the update efficiency between resting state and task-general architecture (Fig. 6). In the brain, it was assumed that the communication between nerve groups was based on coherence. Through coherence, two neural combinations of synchronized activities can exchange information (Fries, 2005; Vasa et al., 2015). The update efficiency of synchrony is a method of isolation and integration. It indicated that during the resting state, the high degree of synchrony between high-order cognitive networks can predict task performance, and network connectivity showed the tendency of integration and separation related to cognitive performance (Alderson, Bokde, Kelso, Maguire, \& Coyle, 2020). The exchange of information between high-order cognitive networks is more flexible, the information exchange of sensorimotor network is more stable, and the ability of switching from resting state to task configuration is stronger.

On the contrary, the changes in synchrony of the task-based RSNs have nothing to do with update efficiency. Brain activity at the resting state when subjects were not performing any explicit task predicted differences in fMRI activation across a range of cognitive paradigms. The resting state functional connectivity thus already contained the repertoire that is then expressed during task-based fMRI (Tavor et al., 2016). The observed fluctuations in network topology during the break are likely to be directly related to ongoing cognitive processing (Shine \& Poldrack, 2018). Current research on RSNs showed that the functional couplings between regions at resting state contain information relevant to cognition, perception and behavior (Sadaghiani \& Kleinschmidt, 2013), rather than simply reflecting invariant structural anatomy, historical coactivation patterns, or internal dynamics of local areas, intrinsic activity predicts subsequent perceptual processing (van den Berg, Appelbaum, Clark, Lorist, \& Woldorff, 2016). 


\subsection{Participants with pre-configuration showed a better performance.}

The update efficiency in brain network organization is positively related to general intelligence and behavior accuracy (Fig. 7), the ability to perform a wide variety of cognitively challenging tasks well. It showed that the ability of participants to answer correct questions inside and outside the scanner is related to their internal neuron dynamics. The update efficiency reflected the difficulty of switching to the task-general architecture from the resting state (Schultz \& Cole, 2016a, 2016b). Specifically, the brain network configuration at rest was already closer to a wide variety of task configurations in intelligent individuals. The ability to modify network connectivity efficiently when task demands change is a hallmark of high intelligence (Cole et al., 2014).

Increasing reasoning demands were supported by the flexible reconfiguration of large-scale functional brain networks (Cocchi et al., 2014), but a recent study has demonstrated that such reconfigurations are relatively modest and occur within a preserved global network architecture(Hearne et al., 2017). Successful cognition was likely contingent on possessing an adequate a priori dynamic configuration before the onset of task-relevant stimuli, as opposed to simple ad hoc adjustments after the fact (Bolt, Anderson, \& Uddin, 2018). Therefore, resting state activity may reflect the brain's predictive engagement with the environment (Sadaghiani \& Kleinschmidt, 2013). Given that the resting state reflects previous experience and the anticipation of likely future events, a RSN architecture 'pre-configured' for task is more in line with future cognitive requirements.

\subsection{The task states synchrony was higher than the resting state synchrony.}

Compared with resting state, the global synchrony during the task is higher, and the synchrony interaction between RSNs is also higher. Compared with the resting state, the co-activation network shows higher global efficiency, smaller average clustering coefficient and lower modularity, which shows that the global information transmission and system during task execution more effective between integrations(Maffei \& Sessa, 
2021). The global integration of the brain increases during the tasks, and as the neural activities of the internal brain system required for task execution become relevant, the functional connections between RSNs become stronger (Cohen \& D'Esposito, 2016).

The change of synchrony is regulated by the needs of cognitive tasks and is a distinctive feature of the continuous activity of the human cortex (Palva, Palva, \& Kaila, 2005). In general, successful behavior depends on effective communication between brain regions. The communication between brain regions can be assessed by analyzing the synchrony. Moreover, the degree of synchrony between regions is more representative of changes in brain function intensity than the analysis of task activation to a certain extent (Hummel \& Gerloff, 2005). And task performance regulates functional interactions in the brain(Li, Graham, \& Werner-Seidler, 2021). When the brain performed a task, it adjusts the brain's connection changes according to the task, exchanges more information, and reconfigures the brain network (Friston, 2011). Therefore, the synchrony is higher during the task.

\section{Conclusion}

We reported a study about the synchrony of healthy young people at resting and task states. Different tasks have a task-general architecture of synchrony. During the tasks, the synchrony was significantly higher, and the synchrony between the high-order cognitive networks is significantly enhanced. It showed that synchrony provides great potential for linking RSNs activities with cognition and behavior. In addition, subjects with high synchrony at resting state have better pre-configurations and show more outstanding cognitive abilities.

\section{Declarations}

\section{Funding}

This project was supported by National Natural Science Functional of China (61873178, 
61876124 and 61906130) and the Shanxi Provincial International Cooperation Foundation (201803D421047).

\section{Conflicts of interest}

None of the authors have a conflict of interest to declare.

\section{Ethics approval}

All participants provided informed consent according to the HCP research ethics committee.

\section{Consent to participate}

Not applicable.

\section{Consent for publication}

All authors have read and agreed to the published version of the manuscript.

\section{Availability of data and material}

These data were derived from the following resources available in the public domain ( https://www.humanconnectome.org/data ).

\section{Code availability}

The code is implemented in a custom script developed in MATLAB 2016b. The code is currently not publicly accessible.

\section{Authors' contributions}

Study design (C.F. and J.W.), data collection or acquisition (L.Y., J.L. .and C.F.), statistical analysis (C.F. and H.G.), interpretation of results (C.F., J.X. and J.W.), drafting the manuscript work or revising it critically for important intellectual content (C.F., H.G., J.W., Y.L. and J.X.) and approval of final version to be published and 
agreement to be accountable for the integrity and accuracy of all aspects of the work

(All authors).

\section{References}

Alderson, T. H., Bokde, A. L. W., Kelso, J. A. S., Maguire, L., \& Coyle, D. (2020). Metastable neural dynamics underlies cognitive performance across multiple behavioural paradigms. Hum Brain Mapp, 41(12), 3212-3234. Retrieved from https://www.ncbi.nlm.nih.gov/pubmed/32301561. doi:10.1002/hbm.25009

Alderson, T. H., Bokde, A. L. W., Kelso, J. A. S., Maguire, L., Coyle, D., \& Alzheimer's Disease Neuroimaging, I. (2018). Metastable neural dynamics in Alzheimer's disease are disrupted by lesions to the structural connectome. Neuroimage, 183, 438-455. Retrieved from https://www.ncbi.nlm.nih.gov/pubmed/30130642. doi:10.1016/j.neuroimage.2018.08.033

Avena-Koenigsberger, A., Misic, B., \& Sporns, O. (2017). Communication dynamics in complex brain networks. Nat Rev Neurosci, 19(1), 17-33. Retrieved from https://www.ncbi.nlm.nih.gov/pubmed/29238085. doi:10.1038/nrn.2017.149

Bakhshayesh, H., Fitzgibbon, S. P., Janani, A. S., Grummett, T. S., \& Pope, K. J. (2019). Detecting synchrony in EEG: A comparative study of functional connectivity measures. Comput Biol Med, 105, 1-15. Retrieved from https://www.ncbi.nlm.nih.gov/pubmed/30562626. doi:10.1016/j.compbiomed.2018.12.005

Barch, D. M., Burgess, G. C., Harms, M. P., Petersen, S. E., Schlaggar, B. L., Corbetta, M., . . . Consortium, W. U.-M. H. (2013). Function in the human connectome: task-fMRI and individual differences in behavior. Neuroimage, 80, 169-189. Retrieved from $\quad$ https://www.ncbi.nlm.nih.gov/pubmed/23684877. doi:10.1016/j.neuroimage.2013.05.033

Bolt, T., Anderson, M. L., \& Uddin, L. Q. (2018). Beyond the evoked/intrinsic neural process dichotomy. Netw Neurosci, 2(1), 1-22. Retrieved from https://www.ncbi.nlm.nih.gov/pubmed/29911670. doi:10.1162/NETN_a_00028

Boring, M. J., Ridgeway, K., Shvartsman, M., \& Jonker, T. R. (2020). Continuous decoding of cognitive load from electroencephalography reveals task-general and task-specific correlates. J Neural Eng, 17(5), 056016. Retrieved from https://www.ncbi.nlm.nih.gov/pubmed/32947265. doi:10.1088/17412552/abb9bc

Bullmore, E., \& Sporns, O. (2012). The economy of brain network organization. Nat Rev Neurosci, 13(5), 336-349. Retrieved from https://www.ncbi.nlm.nih.gov/pubmed/22498897. doi:10.1038/nrn3214

Chan, M. Y., Alhazmi, F. H., Park, D. C., Savalia, N. K., \& Wig, G. S. (2017). RestingState Network Topology Differentiates Task Signals across the Adult Life Span. J Neurosci, 37(10), 2734-2745. Retrieved from 
https://www.ncbi.nlm.nih.gov/pubmed/28174333.

doi:10.1523/JNEUROSCI.2406-16.2017

Cocchi, L., Halford, G. S., Zalesky, A., Harding, I. H., Ramm, B. J., Cutmore, T., . . . Mattingley, J. B. (2014). Complexity in relational processing predicts changes in functional brain network dynamics. Cereb Cortex, 24(9), 2283-2296. Retrieved from https://www.ncbi.nlm.nih.gov/pubmed/23563963. doi:10.1093/cercor/bht075

Cohen, J. R., \& D'Esposito, M. (2016). The Segregation and Integration of Distinct Brain Networks and Their Relationship to Cognition. J Neurosci, 36(48), 12083-12094. Retrieved

from https://www.ncbi.nlm.nih.gov/pubmed/27903719. doi:10.1523/JNEUROSCI.2965-15.2016

Cole, M. W., Bassett, D. S., Power, J. D., Braver, T. S., \& Petersen, S. E. (2014). Intrinsic and task-evoked network architectures of the human brain. Neuron, 83(1), 238-251. Retrieved from https://www.ncbi.nlm.nih.gov/pubmed/24991964. doi:10.1016/j.neuron.2014.05.014

Dajani, D. R., Odriozola, P., Winters, M., Voorhies, W., Marcano, S., Baez, A., . . . Uddin, L. Q. (2020). Measuring Cognitive Flexibility with the Flexible Item Selection Task: From fMRI Adaptation to Individual Connectome Mapping. $J$ Cogn Neurosci, 32(6), 1026-1045. Retrieved from https://www.ncbi.nlm.nih.gov/pubmed/32013686. doi:10.1162/jocn_a_01536

Ferrier, J., Tiran, E., Deffieux, T., Tanter, M., \& Lenkei, Z. (2020). Functional imaging evidence for task-induced deactivation and disconnection of a major default mode network hub in the mouse brain. Proc Natl Acad Sci US A, 117(26), 15270-15280. Retrieved from https://www.ncbi.nlm.nih.gov/pubmed/32541017. doi:10.1073/pnas.1920475117

Freitas, L. G. A., Bolton, T. A. W., Krikler, B. E., Jochaut, D., Giraud, A. L., Huppi, P. S., \& Van De Ville, D. (2020). Time-resolved effective connectivity in task fMRI: Psychophysiological interactions of Co-Activation patterns. Neuroimage, 212, 116635. Retrieved from https://www.ncbi.nlm.nih.gov/pubmed/32105884. doi:10.1016/j.neuroimage.2020.116635

Fries, P. (2005). A mechanism for cognitive dynamics: neuronal communication through neuronal coherence. Trends Cogn Sci, 9(10), 474-480. Retrieved from https://www.ncbi.nlm.nih.gov/pubmed/16150631.

doi:10.1016/j.tics.2005.08.011

Friston, K. J. (2011). Functional and effective connectivity: a review. Brain Connect, 1(1), 13-36. Retrieved from https://www.ncbi.nlm.nih.gov/pubmed/22432952. doi:10.1089/brain.2011.0008

Glasser, M. F., Coalson, T. S., Robinson, E. C., Hacker, C. D., Harwell, J., Yacoub, E., . . Van Essen, D. C. (2016). A multi-modal parcellation of human cerebral cortex. Nature, 536(7615), 171-178. Retrieved from https://www.ncbi.nlm.nih.gov/pubmed/27437579. doi:10.1038/nature18933 
Glasser, M. F., Sotiropoulos, S. N., Wilson, J. A., Coalson, T. S., Fischl, B., Andersson, J. L., . . Consortium, W. U.-M. H. (2013). The minimal preprocessing pipelines for the Human Connectome Project. Neuroimage, 80, 105-124. Retrieved from https://www.ncbi.nlm.nih.gov/pubmed/23668970.

doi:10.1016/j.neuroimage.2013.04.127

Gu, S., Pasqualetti, F., Cieslak, M., Telesford, Q. K., Yu, A. B., Kahn, A. E., . . Bassett, D. S. (2015). Controllability of structural brain networks. Nat Commun, 6, 8414. Retrieved from $\quad$ https://www.ncbi.nlm.nih.gov/pubmed/26423222. doi: $10.1038 /$ ncomms 9414

Gu, S., Satterthwaite, T. D., Medaglia, J. D., Yang, M., Gur, R. E., Gur, R. C., \& Bassett, D. S. (2015). Emergence of system roles in normative neurodevelopment. Proc Natl Acad Sci $U \quad S$ A, 112(44), 13681-13686. Retrieved from https://www.ncbi.nlm.nih.gov/pubmed/26483477. doi:10.1073/pnas.1502829112

Han, S., Zong, X., Hu, M., Yu, Y., Wang, X., Long, Z., . . Chen, H. (2017). Frequencyselective alteration in the resting-state corticostriatal-thalamo-cortical circuit correlates with symptoms severity in first-episode drug-naive patients with schizophrenia. Schizophr Res, 189, 175-180. Retrieved from https://www.ncbi.nlm.nih.gov/pubmed/28236519. doi:10.1016/j.schres.2017.02.019

Hearne, L. J., Cocchi, L., Zalesky, A., \& Mattingley, J. B. (2017). Reconfiguration of Brain Network Architectures between Resting-State and ComplexityDependent Cognitive Reasoning. J Neurosci, 37(35), 8399-8411. Retrieved from https://www.ncbi.nlm.nih.gov/pubmed/28760864. doi:10.1523/JNEUROSCI.0485-17.2017

Hummel, F., \& Gerloff, C. (2005). Larger interregional synchrony is associated with greater behavioral success in a complex sensory integration task in humans. Cereb Cortex, 15(5), 670-678. Retrieved from https://www.ncbi.nlm.nih.gov/pubmed/15342429. doi:10.1093/cercor/bhh170

Ito, T., Kulkarni, K. R., Schultz, D. H., Mill, R. D., Chen, R. H., Solomyak, L. I., \& Cole, M. W. (2017). Cognitive task information is transferred between brain regions via resting-state network topology. Nat Commun, 8(1), 1027. Retrieved from https://www.ncbi.nlm.nih.gov/pubmed/29044112. doi:10.1038/s41467017-01000-w

Kringelbach, M. L., \& Deco, G. (2020). Brain States and Transitions: Insights from Computational Neuroscience. Cell Rep, 32(10), 108128. Retrieved from https://www.ncbi.nlm.nih.gov/pubmed/32905760. doi:10.1016/j.celrep.2020.108128

Li, J., Bolt, T., Bzdok, D., Nomi, J. S., Yeo, B. T. T., Spreng, R. N., \& Uddin, L. Q. (2019). Topography and behavioral relevance of the global signal in the human brain. Sci Rep, 9(1), 14286. Retrieved from https://www.ncbi.nlm.nih.gov/pubmed/31582792. doi:10.1038/s41598-01950750-8

Li, M., Han, Y., Aburn, M. J., Breakspear, M., Poldrack, R. A., Shine, J. M., \& Lizier, 
J. T. (2019). Transitions in information processing dynamics at the whole-brain network level are driven by alterations in neural gain. PLoS Comput Biol, 15(10), e1006957. Retrieved from https://www.ncbi.nlm.nih.gov/pubmed/31613882. doi:10.1371/journal.pcbi.1006957

Li, S. H., Graham, B. M., \& Werner-Seidler, A. (2021). Gender Differences in Adolescent Sleep Disturbance and Treatment Response to Smartphone AppDelivered Cognitive Behavioral Therapy for Insomnia: Exploratory Study. JMIR Form Res, 5(3), e22498. Retrieved from https://www.ncbi.nlm.nih.gov/pubmed/33755029. doi:10.2196/22498

Maffei, A., \& Sessa, P. (2021). Event-related network changes unfold the dynamics of cortical integration during face processing. Psychophysiology, 58(5), e13786. Retrieved from https://www.ncbi.nlm.nih.gov/pubmed/33550632. doi:10.1111/psyp.13786

Markett, S., Jawinski, P., Kirsch, P., \& Gerchen, M. F. (2020). Specific and segregated changes to the functional connectome evoked by the processing of emotional faces: A task-based connectome study. Sci Rep, 10(1), 4822. Retrieved from https://www.ncbi.nlm.nih.gov/pubmed/32179856. doi:10.1038/s41598-02061522-0

Meijer, K. A., Eijlers, A. J. C., Douw, L., Uitdehaag, B. M. J., Barkhof, F., Geurts, J. J. G., \& Schoonheim, M. M. (2017). Increased connectivity of hub networks and cognitive impairment in multiple sclerosis. Neurology, 88(22), 2107-2114. Retrieved from $\quad$ https://www.ncbi.nlm.nih.gov/pubmed/28468841. doi:10.1212/WNL.0000000000003982

Messel, M. S., Raud, L., Hoff, P. K., Skaftnes, C. S., \& Huster, R. J. (2019). Strategy switches in proactive inhibitory control and their association with task-general and stopping-specific networks. Neuropsychologia, 135, 107220. Retrieved from https://www.ncbi.nlm.nih.gov/pubmed/31586553. doi:10.1016/j.neuropsychologia.2019.107220

Millar, P. R., Ances, B. M., Gordon, B. A., Benzinger, T. L. S., Morris, J. C., \& Balota, D. A. (2021). Evaluating Cognitive Relationships with Resting-State and Taskdriven Blood Oxygen Level-Dependent Variability. J Cogn Neurosci, 33(2), 279-302. Retrieved from https://www.ncbi.nlm.nih.gov/pubmed/33135966. doi:10.1162/jocn_a_01645

Min, W., Liu, J., \& Zhang, S. (2018). Edge-group sparse PCA for network-guided high dimensional data analysis. Bioinformatics, 34(20), 3479-3487. Retrieved from https://www.ncbi.nlm.nih.gov/pubmed/29726900. doi:10.1093/bioinformatics/bty362

Palmigiano, A., Geisel, T., Wolf, F., \& Battaglia, D. (2017). Flexible information routing by transient synchrony. Nat Neurosci, 20(7), 1014-1022. Retrieved from https://www.ncbi.nlm.nih.gov/pubmed/28530664. doi:10.1038/nn.4569

Palva, J. M., Palva, S., \& Kaila, K. (2005). Phase synchrony among neuronal oscillations in the human cortex. J Neurosci, 25(15), 3962-3972. Retrieved from https://www.ncbi.nlm.nih.gov/pubmed/15829648.

doi:10.1523/JNEUROSCI.4250-04.2005 
Petersen, A., Zhao, J., Carmichael, O., \& Muller, H. G. (2016). Quantifying Individual Brain Connectivity with Functional Principal Component Analysis for Networks. Brain Connect, 6(7), 540-547. Retrieved from https://www.ncbi.nlm.nih.gov/pubmed/27267074. doi:10.1089/brain.2016.0420

Ponce-Alvarez, A., Deco, G., Hagmann, P., Romani, G. L., Mantini, D., \& Corbetta, M. (2015). Resting-state temporal synchronization networks emerge from connectivity topology and heterogeneity. PLoS Comput Biol, 11(2), e1004100. Retrieved from https://www.ncbi.nlm.nih.gov/pubmed/25692996. doi:10.1371/journal.pcbi.1004100

Raichle, M. E. (2010). Two views of brain function. Trends Cogn Sci, 14(4), 180-190. Retrieved from https://www.ncbi.nlm.nih.gov/pubmed/20206576. doi:10.1016/j.tics.2010.01.008

Sadaghiani, S., \& Kleinschmidt, A. (2013). Functional interactions between intrinsic brain activity and behavior. Neuroimage, 80, 379-386. Retrieved from https://www.ncbi.nlm.nih.gov/pubmed/23643921.

doi:10.1016/j.neuroimage.2013.04.100

Schultz, D. H., \& Cole, M. W. (2016a). Higher Intelligence Is Associated with Less Task-Related Brain Network Reconfiguration. J Neurosci, 36(33), 8551-8561. Retrieved from https://www.ncbi.nlm.nih.gov/pubmed/27535904. doi:10.1523/JNEUROSCI.0358-16.2016

Schultz, D. H., \& Cole, M. W. (2016b). Integrated Brain Network Architecture Supports Cognitive Task Performance. Neuron, 92(2), 278-279. Retrieved from https://www.ncbi.nlm.nih.gov/pubmed/27764661. doi:10.1016/j.neuron.2016.10.004

Shine, J. M., \& Poldrack, R. A. (2018). Principles of dynamic network reconfiguration across diverse brain states. Neuroimage, 180(Pt B), 396-405. Retrieved from https://www.ncbi.nlm.nih.gov/pubmed/28782684. doi:10.1016/j.neuroimage.2017.08.010

Skardal, P. S., \& Arenas, A. (2020). Higher order interactions in complex networks of phase oscillators promote abrupt synchronization switching. Communications Physics, 3(1). Retrieved from <Go to ISI >://WOS:000596087100001. doi:ARTN 218

10.1038/s42005-020-00485-0

Tavor, I., Parker Jones, O., Mars, R. B., Smith, S. M., Behrens, T. E., \& Jbabdi, S. (2016). Task-free MRI predicts individual differences in brain activity during task performance. Science, 352(6282), 216-220. Retrieved from https://www.ncbi.nlm.nih.gov/pubmed/27124457. doi:10.1126/science.aad8127

Ugurbil, K., Xu, J., Auerbach, E. J., Moeller, S., Vu, A. T., Duarte-Carvajalino, J. M., .. . Consortium, W. U.-M. H. (2013). Pushing spatial and temporal resolution for functional and diffusion MRI in the Human Connectome Project. Neuroimage, 80, 80-104. Retrieved from https://www.ncbi.nlm.nih.gov/pubmed/23702417. doi:10.1016/j.neuroimage.2013.05.012 
van den Berg, B., Appelbaum, L. G., Clark, K., Lorist, M. M., \& Woldorff, M. G. (2016). Visual search performance is predicted by both prestimulus and poststimulus electrical brain activity. Sci Rep, 6, 37718. Retrieved from https://www.ncbi.nlm.nih.gov/pubmed/27901053. doi:10.1038/srep37718

Van Essen, D. C., Smith, S. M., Barch, D. M., Behrens, T. E., Yacoub, E., Ugurbil, K., \& Consortium, W. U.-M. H. (2013). The WU-Minn Human Connectome Project: an overview. Neuroimage, 80, 62-79. Retrieved from https://www.ncbi.nlm.nih.gov/pubmed/23684880. doi:10.1016/j.neuroimage.2013.05.041

Vasa, F., Shanahan, M., Hellyer, P. J., Scott, G., Cabral, J., \& Leech, R. (2015). Effects of lesions on synchrony and metastability in cortical networks. Neuroimage, 118, 456-467. Retrieved from https://www.ncbi.nlm.nih.gov/pubmed/26049146. doi:10.1016/j.neuroimage.2015.05.042

Zhang, W., Tang, F., Zhou, X., \& Li, H. (2020). Dynamic Reconfiguration of Functional Topology in Human Brain Networks: From Resting to Task States. Neural Plast, 2020 , 8837615. Retrieved from https://www.ncbi.nlm.nih.gov/pubmed/32963519. doi:10.1155/2020/8837615 


\section{Figures:}

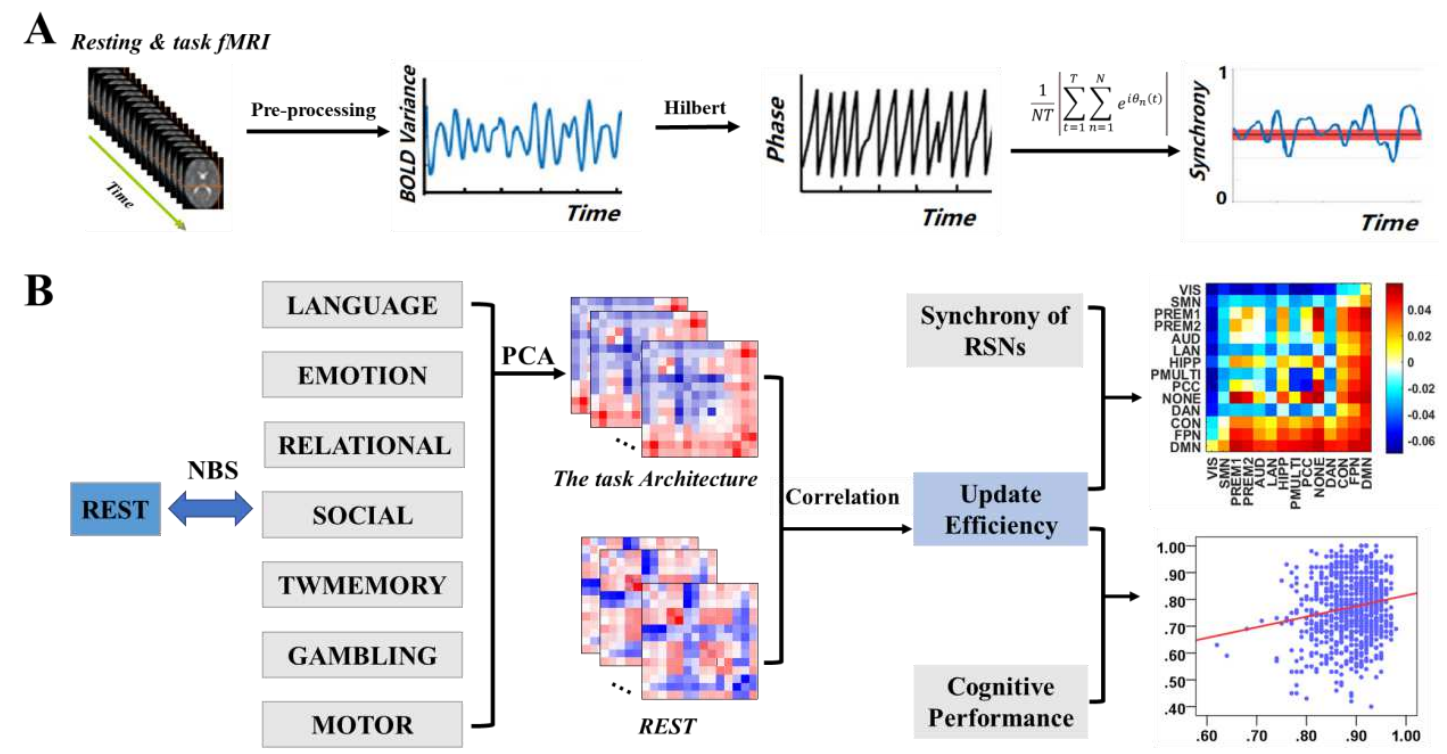

Fig.1 Overview of experimental design. A. Data pre-processing and calculate synchrony. B. Relationship between resting state and task states. 


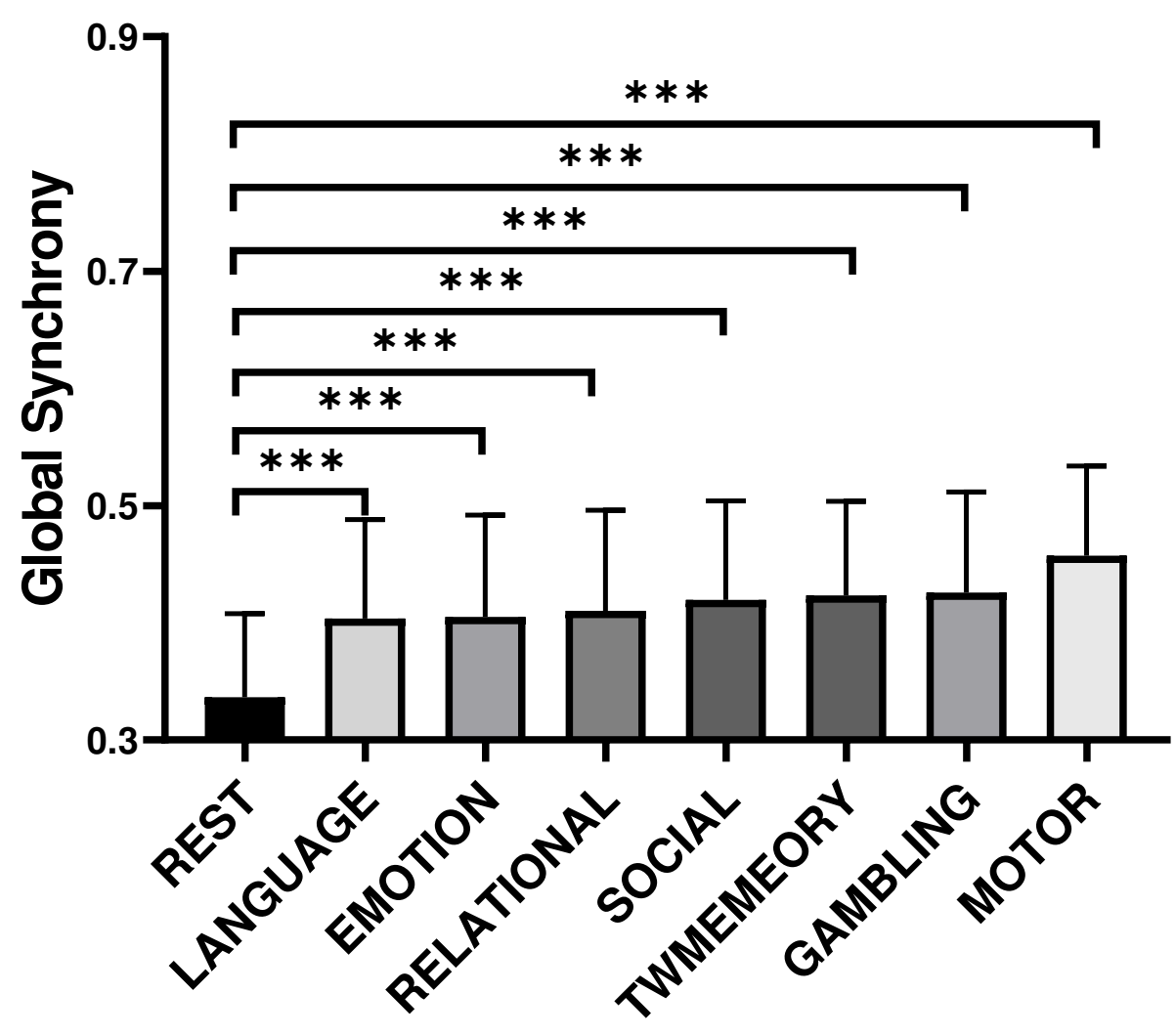

Fig. 2 The difference of global synchrony during resting state and different task states. Bars display the mean value, $95 \% \mathrm{CI}$ and one $\mathrm{SD}$ with individual subjects indicated. Tasks arranged in ascending order of mean synchrony. 


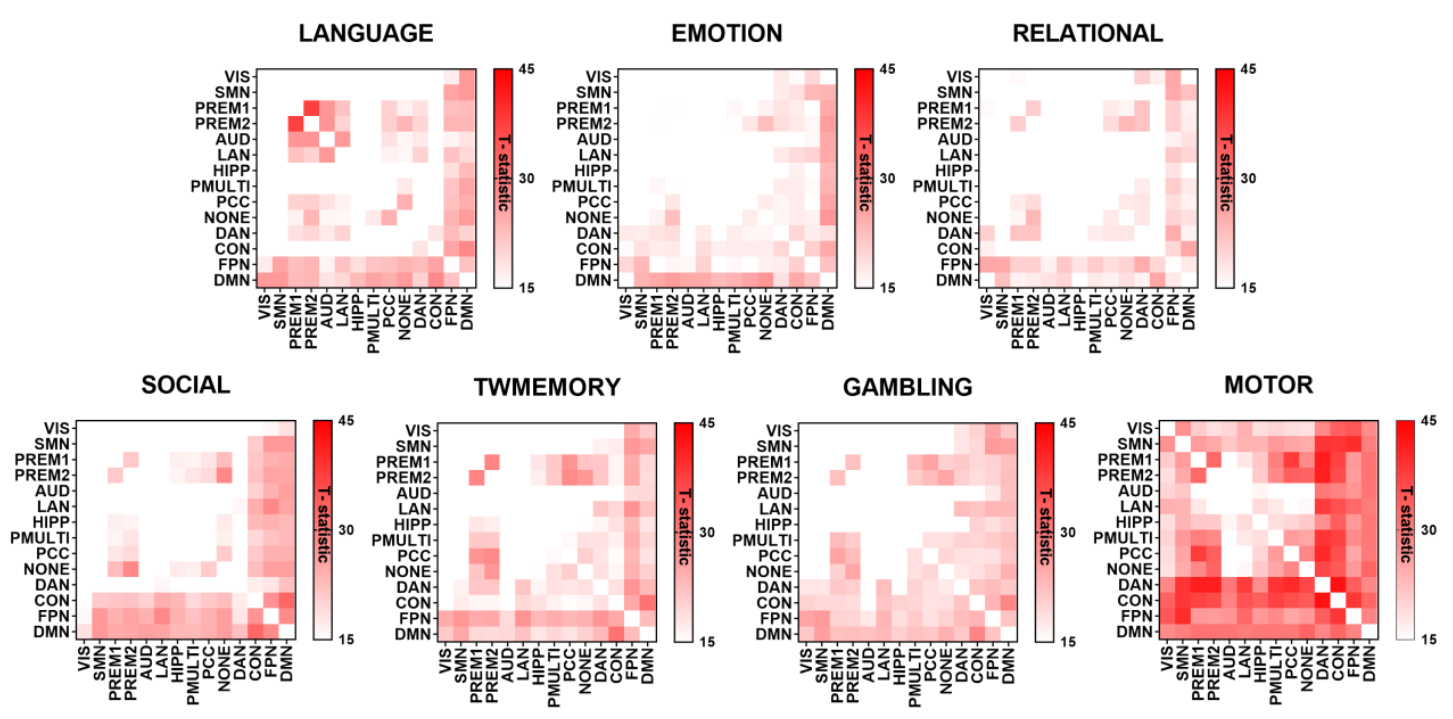

Fig. 3 The difference of synchrony between RSNs in resting state and different task states. The graph used the t-value to show the largest connected subgraph of the difference. The darker the color, the greater the difference. 
A

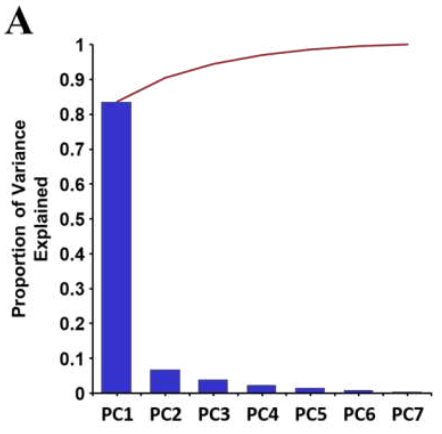

B

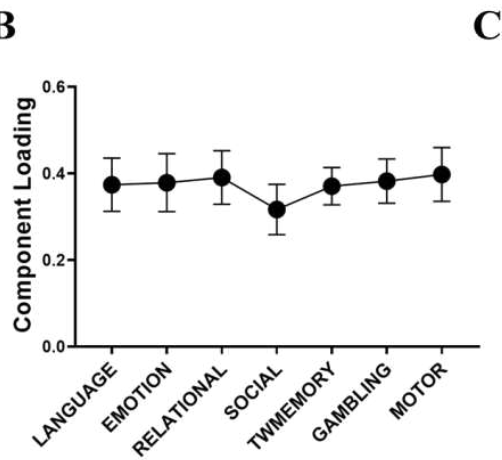

C

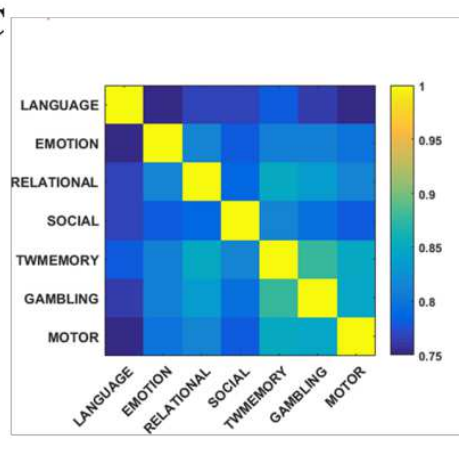

Fig. 4 PCA quantifies the degree of sharing based on the functional configuration of seven tasks. A. The histogram of each component accounts for the variance between the seven tasks, and the broken line indicates the cumulative proportion of each component. B. The loading of seven tasks. The error bar indicates the standard deviation. C. Correlation between the synchrony of the seven tasks. 


\section{A Low Synchrony Subnet}

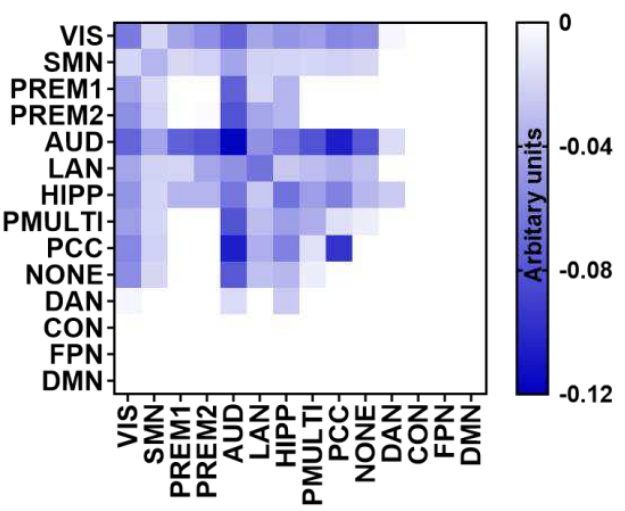

B High Synchrony Subnet

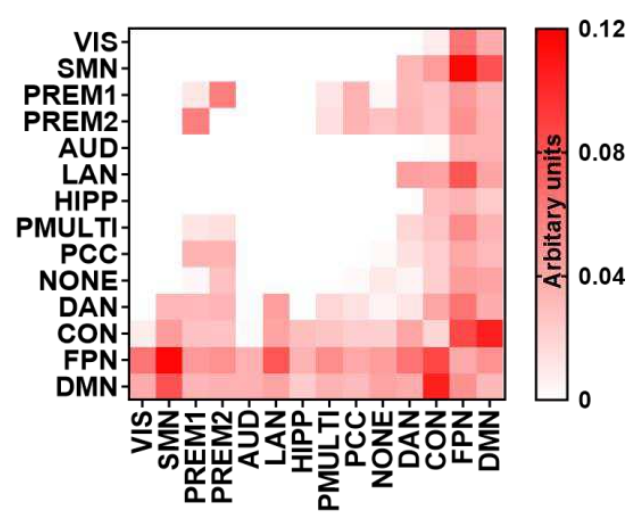

Smaller increases from rest

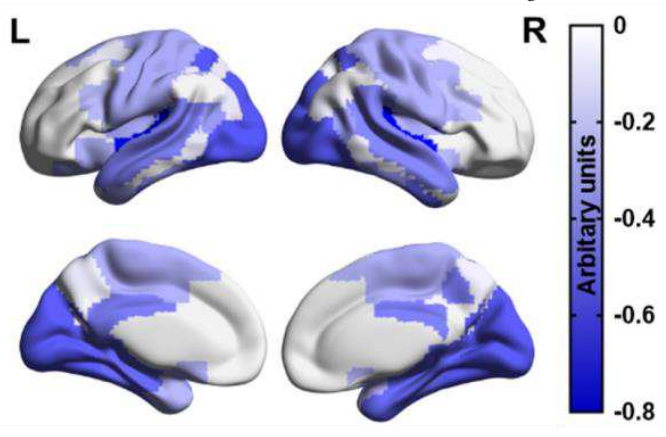

Larger increases from rest
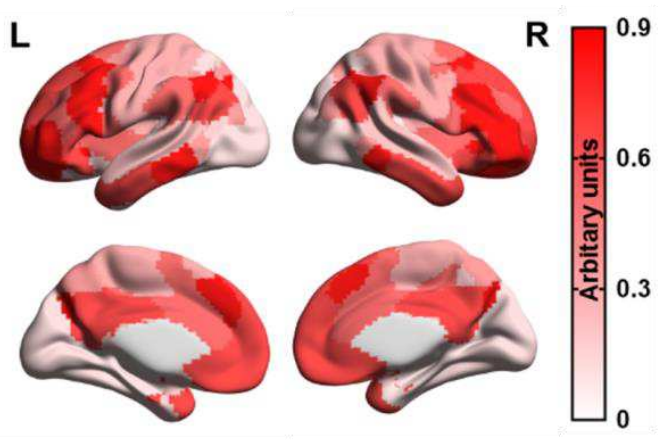

Fig. 5 PCA reveals a task-general network architecture of synchrony. A. Low synchrony subnet. B. High synchrony subnet. 
A

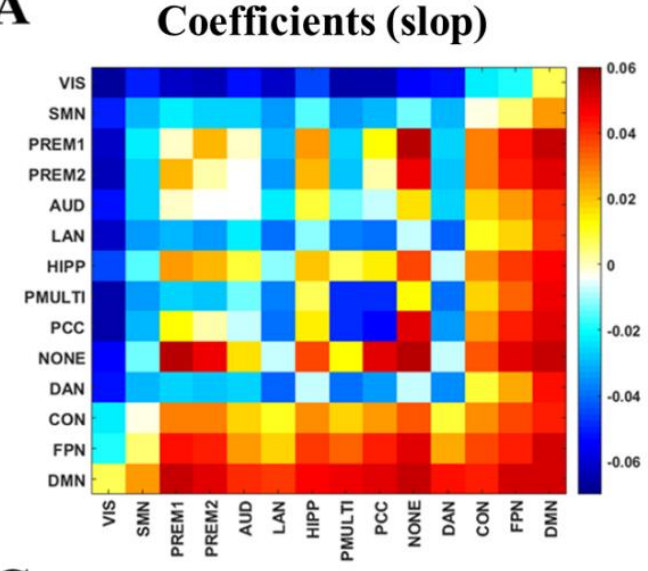

C

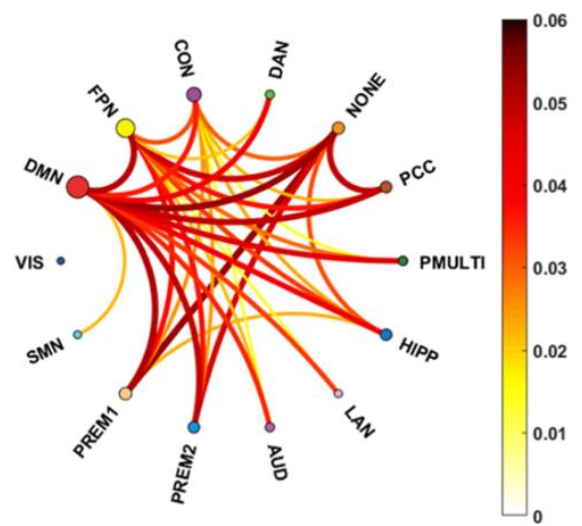

B

$\operatorname{FDR}(p<0.01)$

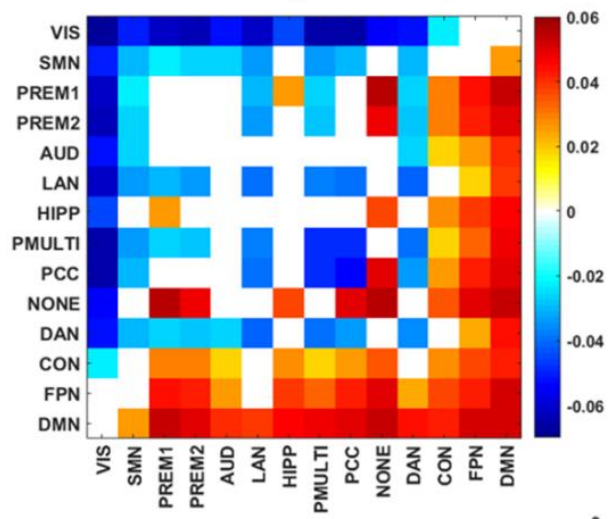

D

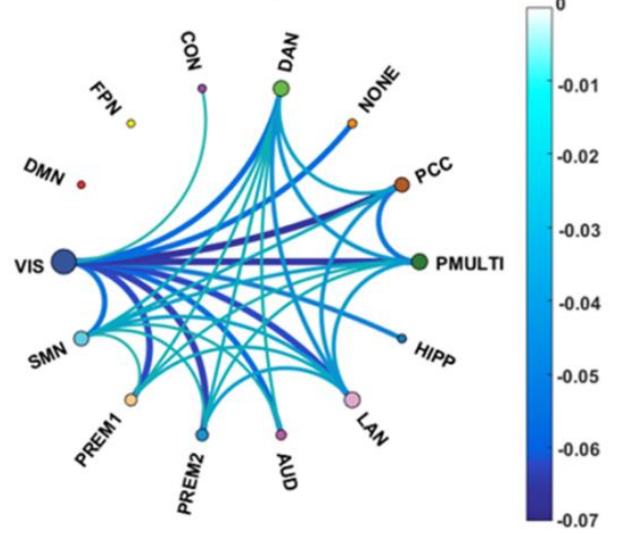

Fig. 6 The correlation between the synchrony of the RSNs and the update efficiency. A. The correlation coefficient (slope) between synchrony of RSNs and update efficiency. B. There is a significant correlation coefficient between synchrony of RSNs and update efficiency. C. There are RSNs with positive correlation between update efficiency and synchrony. The darker the color, the higher the correlation. The size of the node represents the sum of the correlations at the RSN. D. There are RSNs with negative correlation between update efficiency and synchrony. 

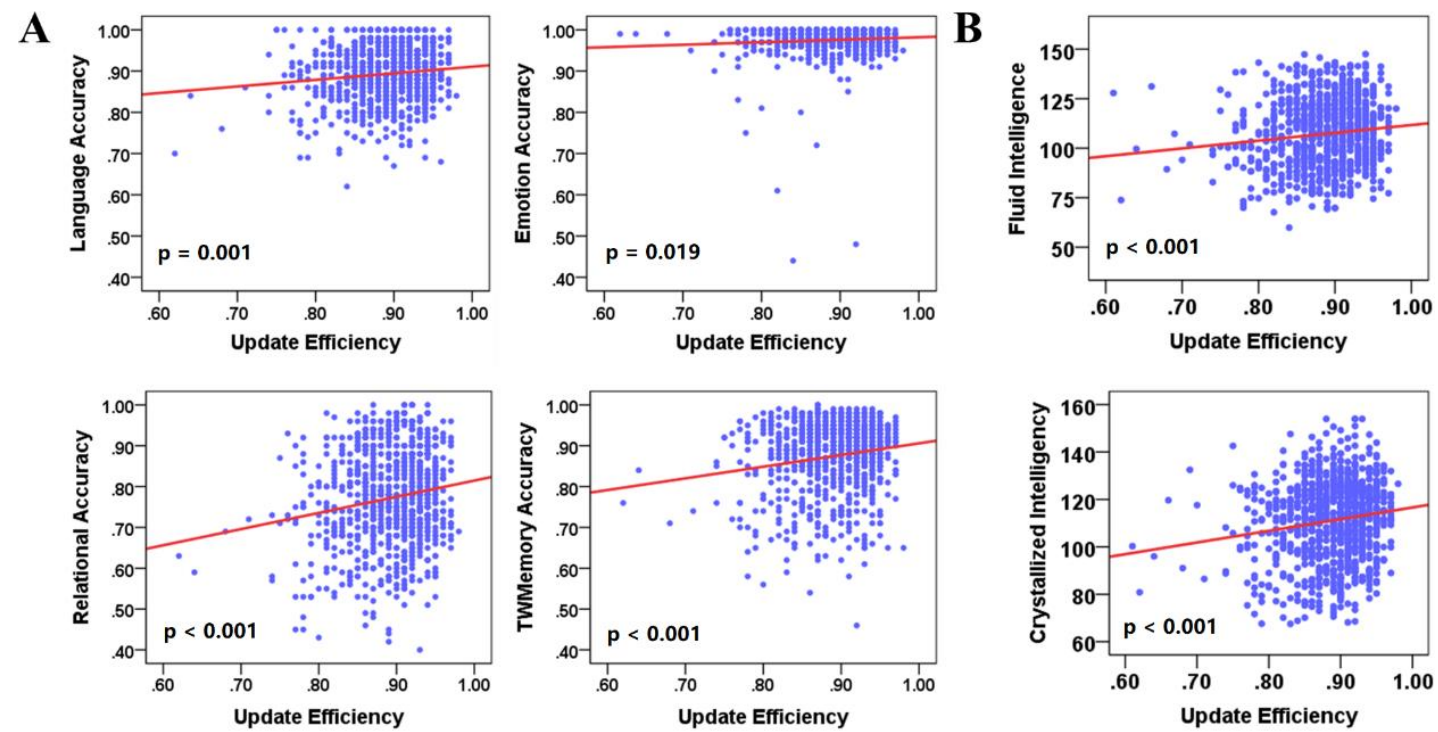

Fig. 7 The correlation between update efficiency and cognitive performance. A. The correlation between update efficiency and behavioral accuracy. B. The correlation between update efficiency and cognitive intelligence. There is a positive correlation between update efficiency and behavioral accuracy. 


\section{Tables:}

Table 1 Basic information of the participants

\begin{tabular}{ll}
\hline Characteristic & Value \\
\hline Gender (Male : Female) & $503: 451$ \\
Age (Years) & $22-35$ \\
Language accuracy & $80.03 \pm 6.96$ \\
Emotion accuracy & $97.50 \pm 3.65$ \\
Relational accuracy & $76.26 \pm 12.49$ \\
Working Memory accuracy & $87.79 \pm 8,79$ \\
Fluid intelligence & $107.31 \pm 16.60$ \\
Crystallized intelligence & $111.10 \pm 16.61$ \\
\hline
\end{tabular}


Table 2 Seven human connectome project fMRI tasks

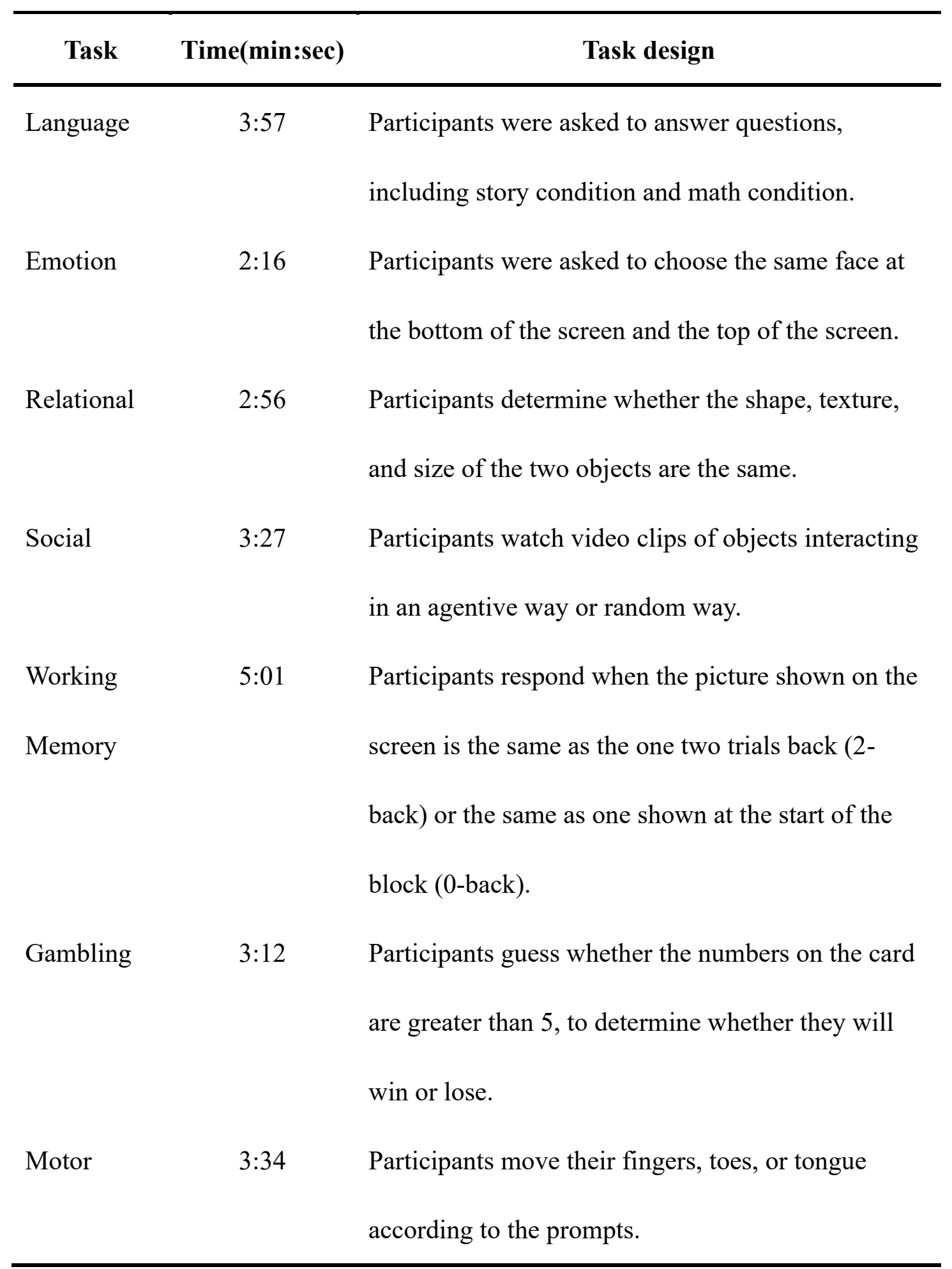




\section{Supplementary Files}

This is a list of supplementary files associated with this preprint. Click to download.

- BIBChecklist.docx 